\title{
The engineered tetra-valent antibody, ASP4021, fully activates the Tie2 receptor with comparable potency to its natural ligand, angiopoietin-1
}

\section{Yukari Koya}

Astellas Pharma (Japan)

Hiromi Nara

Astellas Pharma (Japan)

Shigenori Yagi

Astellas Pharma (Japan)

Chihoko Ueno

Astellas Pharma (Japan)

Masazumi Kamohara ( $\sim$ masazumi.kamohara@astellas.com )

Astellas Pharma (Japan)

\section{Research Article}

Keywords: ASP4021, Tie2 receptor, novel engineered antibody

Posted Date: February 26th, 2021

DOl: https://doi.org/10.21203/rs.3.rs-244826/v1

License: (c) (1) This work is licensed under a Creative Commons Attribution 4.0 International License.

Read Full License

Version of Record: A version of this preprint was published at Scientific Reports on July 7th, 2021. See the published version at https://doi.org/10.1038/s41598-021-93660-4. 


\section{Title page}

The engineered tetra-valent antibody, ASP4021, fully activates the Tie2 receptor with comparable

potency to its natural ligand, angiopoietin-1

Yukari Koya, Hiromi Nara, Shigenori Yagi, Chihoko Ueno, and Masazumi Kamohara*

Drug Discovery Research, Astellas Pharma Inc., Tsukuba, Japan

Correspondence should be addressed to M.K. (masazumi.kamohara@astellas.com) 


\section{Introductory paragraph}

Activation of the tyrosine kinase with Ig and epidermal growth factor homology domain 2

(Tie2) receptor by angiopoietin-1 (Ang1) is critical for vascular stabilization: it promotes survival signal transduction via auto-phosphorylation and reduces vascular permeability by strengthening tight

junctions between endothelial cells. Thus, Tie2/Ang1 signaling is a promising therapeutic target for vascular diseases. However, in vivo use of existing Tie2 signaling modulators, such as recombinant Ang1, is restricted by limitations in manufacturability and stability. Here, we present a novel engineered tetra-valent agonistic antibody, ASP4021, which can specifically and fully activate the Tie2 receptor in an equivalent manner to Ang1. ASP4021 induced Tie2 self-phosphorylation and inhibited apoptosis in a human primary endothelial cell line. Additionally, single administration of ASP4021 significantly suppressed mustard-oil-induced vascular permeability in rats. ASP4021 may thus be a potential therapeutic candidate for diseases associated with vascular weakness such as diabetic retinopathy, diabetic macular edema and critical limb ischemia. 


\section{Main text}

Tie2 is mainly expressed in vascular endothelial cells and Ang1, its natural ligand, is a

multimer-type-secreted glycoprotein expressed in pericytes. When Ang1 binds Tie2, Tie2 undergoes

oligomerization and auto-phosphorylation, which leads to promotion of anti-apoptotic activity in

vascular endothelial cells via induction of Akt phosphorylation ${ }^{1}$, inhibition of vascular permeability

via suppression of Src signaling ${ }^{2}$, and activation of vascular maturation and vascular remodeling ${ }^{3,4}$.

Furthermore, activation of Tie2 in vascular endothelial cells by Ang1 induces vasodilation and

enhances blood flow via nitric oxide production ${ }^{5}$. Tie2/Ang1 signaling is thus a promising

therapeutic target for diseases associated with vascular weakness such as diabetic retinopathy ${ }^{6-8}$ and

critical limb ischemia ${ }^{9,10}$. Despite the promise of some therapeutic candidates for vascular stability,

however, clinical use of recombinant Ang1 and its variants, such as the chimeric protein COMP-

Ang1, are limited due to difficulties with large scale production, short in vivo half-lives and potential immunogenicity ${ }^{11-13}$.

Agonistic antibodies are an effective alternative to native ligands because technologies for mass production of antibodies are well established. Antibodies are highly stable with long half-lives in circulation and bind specifically to their target protein. The Tie 2 agonistic mouse monoclonal antibody 15B8 induces phosphorylation of the Tie2 receptor and promotes survival and angiogenesis 
of endothelial cells in vitro and in vivo ${ }^{14}$. Furthermore, the Tie 2 agonistic fully human antibody $1-4 \mathrm{~h}$ facilitates phosphorylation of Tie2 and intracellular signal transmission ${ }^{15}$. While 1-4h also increases the formation of capillary-like tubes in endothelial cells in vitro, its effects in an in vivo animal model could not be assessed due to a lack of cross-reactivity for rodent Tie2.

Here, we identified agonistic monoclonal antibodies with comparable activity to that of Ang1. Surprisingly, only dimer and higher molecular weight antibodies, but not monomers, showed agonistic activity in Tie2-expressing $\mathrm{Ba} / \mathrm{F} 3$ cells. We thus hypothesized that tetra-valency might be important for inducing full agonistic activity by facilitating Tie2 receptor dimerization or multimerization for subsequent auto-phosphorylation. As expected, an engineered tetra-valent antibody, but not a general bivalent antibody, showed agonistic and anti-apoptotic activity in Tie2expressing $\mathrm{Ba} / \mathrm{F} 3$ cells. Moreover, single subcutaneous administration of the tetra-valent antibody significantly suppressed mustard-oil-induced vascular permeability in rats in vivo. These results proved our hypothesis, indicating that our engineered tetra-valent antibody is an effective substitute for Ang1 in therapeutic applications. 
First, we immunized VelocImmune mice with a recombinant human Tie2-Fc chimeric protein. Using a standard method, lymphocytes from immunized mice were fused with mouse-derived myeloma cells to generate hybridomas that produce anti-Tie2 antibodies. To select antibodies that bind to the natural conformation of the Tie2 receptor, cell-based ELISA was performed using Tie2expressing $\mathrm{CHO}$ cells. Monoclonal antibodies bind to human Tie2 with cross-reactivity for monkey, rat, and mouse Tie2 were selected using cell-based ELISA with CHO cells expressing Tie2 from each species. Second, to screen for antibodies with natural ligand-like properties, a competitive binding assay was conducted using human Tie2-expressing $\mathrm{CHO}$ cells with labeled Ang1. Antibodies that compete against COMP-Ang1 likely bind to a similar binding site to Ang1 on Tie2. Additionally, because Ang1 and Ang2, a natural antagonist of Tie2, have the same affinity for the same binding site on Tie $2^{16}$, selected antibodies should also show competitive activity against Ang2. Third, to identify a functionally agonistic antibody, agonistic activity was evaluated using the human Tie2-expressing mouse pro-B cell line $\mathrm{Ba} / \mathrm{F} 3$. While $\mathrm{Ba} / \mathrm{F} 3$ depends on exogenous interleukin-3 (IL-3) for survival, this IL-3-dependency can be compensated by ectopic overexpression of a ligand-stimulated or constitutively active tyrosine kinase. The survival of Tie2-expressing $\mathrm{Ba} / \mathrm{F} 3$, evaluated here using a CellTiter-Glo assay, depends on Tie2 agonistic stimulation in the absence of IL-3. While the monoclonal antibody clone 2-16 showed comparable agonistic activity to recombinant Ang1, 
monoclonal antibody clone 2-8 did not (Fig. 1A). Interestingly, some purified antibodies showed unstable and transient agonistic activity that disappeared after a couple of weeks. To examine these intriguing findings, size exclusion chromatography (SEC) analysis was performed, because it is known that some antibody aggregates during purification of antibody using a Protein-A/G column ${ }^{17}$. We observed monomers, dimers and higher molecular weight (HMW) proteins in the purified antibody solutions (Fig. 1B). Surprisingly, monomer-rich fractions, especially fractions $2-4$, showed weak agonistic activity, whereas those rich in dimer and HMW proteins (fractions 5 and 6) showed higher agonistic activity (Fig. 1C). Next, we evaluated the agonistic activity in three fractions containing monomers, dimers and HMW proteins from antibody clone 2-16 (Fig. 1D). While the dimer and HMW fractions showed full agonistic activity equivalent to native Ang1, the monomer fraction only showed partial activity. This suggests that antibodies with four or higher valence acquired agonistic activity through Tie2 multimerization.

We hypothesized that this tetra-valency might be important for full agonistic activity by facilitating Tie2 receptor dimerization or multimerization for subsequent auto-phosphorylation. To produce antibodies with full agonistic activity, anti-Tie2 antibody clones were genetically engineered to form tetra-valent human antibodies. The tetra-valent antibody consisted of two heavy chains and four light chains (Fig. 2A). Each heavy chain comprised two structures, one with a heavy chain 
variable region $(\mathrm{VH})$ followed by a $\mathrm{CH} 1$ region and the other with $\mathrm{VH}, \mathrm{CH} 1, \mathrm{CH} 2$ and $\mathrm{CH} 3$ regions of the human IgG1 heavy chain gene, joined by a linker. Each light chain comprised a variable region (VL) and constant region (CL) of the human immunoglobulin kappa light chain gene. For linker optimization, the IgG1 upper hinge sequence-based linker EPKSCGS, IgG3 upper hinge sequencebased linker ELKTPLGDTTHTGS and IgG3 upper hinge sequence-based long linker ELKTPLGDTTHT(GGGGS) $\times 10$ were prepared. Because all three showed equivalent agonistic efficacy, EPKSCGS was adopted. The engineered tetra-valent human antibody generated from clone 2-16 was called TIE-1-Ig 1 -WT, and the bivalent human antibody was called 2-16A2. Amino acid mutations of L234A, L235A and P331S were introduced into the heavy chain constant region to reduce antibody-dependent cellular cytotoxicity or complement-dependent cytotoxicity because the target molecule, Tie2, is a cell surface antigen ${ }^{18-21}$. The engineered tetra-valent antibody generated from clone 2-16 with mutations in the heavy chain was called ASP4021. The tetra-valent antibodies could be stably produced using the 293 or $\mathrm{CHO}$ cell systems, and purified using affinity chromatography and SEC (Fig. 2B). While both tetra-valent TIE-1-Ig 1 1-WT and ASP4021 showed similarly potent agonistic activity to Ang1 in human Tie2-expressing $\mathrm{Ba} / \mathrm{F} 3$, bivalent 2-16A2 (purified as a monomer) showed weak activity (Fig. 2C). This result proved our hypothesis that genetically engineered tetravalent antibodies, TIE-1-Ig $1-\mathrm{WT}$ and ASP4021, can induce similarly potent agonistic activity to the 
natural ligand Ang1.

To evaluate whether ASP4021 induces anti-apoptotic activity, the Caspase-Glo 3/7 assay was used to evaluate caspase 3/7 activity in Tie2-expressing Ba/F3. ASP4021 showed comparable anti-apoptotic activity to Ang1 (Fig. 2D). To validate the agonistic activity in human primary cells, Tie2 phosphorylation was evaluated in human umbilical vein endothelial cells (HUVEC). Levels of pTie2 after treatment with ASP4021 at $100 \mathrm{ng} / \mathrm{mL}(0.4 \mathrm{nM})$ increased 7.1-fold compared to vehicle control (Fig. 2E). Ang1 induced a maximum 7.0-fold increase in pTie2 at $0.18 \mathrm{nM}(10 \mathrm{ng} / \mathrm{mL})$. Thus, ASP4021 induces anti-apoptotic activity through phosphorylation of Tie2 in $\mathrm{Ba} / \mathrm{F} 3$ cells and human primary cells in the same manner as Ang1.

Before initiating an in vivo study, the cross-reactivity of ASP4021 for monkey, rat and mouse Tie2 was assessed. The binding activity of ASP4021 to recombinant Tie2-Fc protein from four species (including humans) was analyzed using a Biacore system based on a surface plasmon resonance technique. The dissociation constant $\left(\mathrm{K}_{\mathrm{D}}\right)$ for the interaction between ASP4021 and human, mouse, rat and monkey Tie2 was $1.5 \times 10^{-9} \mathrm{~mol} / \mathrm{L}$ [ $95 \%$ confidence interval $(95 \% \mathrm{CI}): 1.0 \times 10^{-9}$ to 2.2 $\left.\times 10^{-9} \mathrm{~mol} / \mathrm{L}\right], 1.0 \times 10^{-9} \mathrm{~mol} / \mathrm{L}\left(95 \% \mathrm{CI}: 6.5 \times 10^{-10}\right.$ to $\left.1.6 \times 10^{-9} \mathrm{~mol} / \mathrm{L}\right), 1.3 \times 10^{-9} \mathrm{~mol} / \mathrm{L}(95 \% \mathrm{CI}: 8.1$ $\times 10^{-10}$ to $\left.2.2 \times 10^{-9} \mathrm{~mol} / \mathrm{L}\right)$, and $1.5 \times 10^{-9} \mathrm{~mol} / \mathrm{L}\left(95 \% \mathrm{CI}: 6.1 \times 10^{-10}\right.$ to $\left.3.8 \times 10^{-9} \mathrm{~mol} / \mathrm{L}\right)$, respectively. ASP4021 showed equivalent and substantially high binding activity to Tie2 from all four species. 
Additionally, binding specificity was checked by comparing with binding to Tie1, another Ang1 receptor. Direct ELISA showed that ASP4021 did not bind to recombinant human Tie1 (Fig. 3A). Pharmacokinetic analysis, conducted to measure the plasma concentration after subcutaneous administration to rats, (Fig. 3B) showed that ASP4021 was stable in blood for more than one week. When the blood concentration fell below around $1 \mu \mathrm{g} / \mathrm{mL}$, ASP4021 was rapidly eliminated from plasma and showed non-linear pharmacokinetics. We predict that the non-linear pharmacokinetics may be explained by target-mediated drug disposition, which is common for monoclonal antibody drugs, especially those targeting membrane receptors ${ }^{22}$.

Finally, we evaluated the antibodies in an in vivo mustard oil-induced vascular permeability rat model, which is the modified Miles assay ${ }^{23}$. In the previous study, Ang1 showed the inhibitory activity on vascular hyper-permeability ${ }^{24}$. First, we compared tetra-valent TIE-1-Ig $\gamma 1-$ WT and bivalent 2-16A2 with vehicle control. The antibodies were subcutaneously administered at $0.3 \mathrm{mg} / \mathrm{kg}$ 48 hours before mustard oil or mineral oil application to the rats' ears. Evans blue dye was systemically administered just before oil application to measure vascular permeability. The amount of leaked Evans blue dye extracted from ears 30 minutes after oil application was measured. As shown in Fig 3C, compared to the vehicle group, the tetra-valent antibody significantly inhibited dye leakage, while bivalent 2-16A2 did not. The blood concentration of 2-16A2 and TIE-1-Ig $\gamma 1-W T 48$ hours after 
administration, the time point at which vascular permeability was evaluated, was $1.3 \pm 0.05 \mathrm{nM}$ and $0.03 \pm 0.003 \mathrm{nM}$, respectively. Based on estimations from the agonistic assay using Tie2-expressing $\mathrm{Ba} / \mathrm{F} 3$ (Fig 2C), the concentration of each antibody was sufficient to exert agonistic activity.

Evaluation of ASP4021 in this model showed that ASP4021 dose-dependently inhibited vascular hyper-permeability (Fig 3D). It was not possible for us to simultaneously compare the efficacy of ASP4021 and Ang1 due to the short half-life of Ang1 in vivo. ASP4021 showed approximately $80 \%$ inhibition of vascular hyper-permeability compared to vehicle, which is higher than that shown by an Ang1-expressing adenovirus (50\% inhibition) in this model ${ }^{24}$. 
Our results demonstrate that dimer and higher multimeric antibodies, but not monomer

antibodies, show agonistic activity to Tie2. Interaction of Tie2 with highly oligomeric Ang1 leads to receptor oligomerization and activation ${ }^{25}$. Although it is not fully understood how ligand bindinginduced oligomerization of Tie2 leads to receptor activation, Ang1-induced Tie2 oligomerization is thought to be critical for activating Tie2 via auto-phosphorylation and initiating downstream signaling. However, purified dimer and higher multimeric antibody fractions are generally unstable, and are therefore unsuitable for therapeutic use in vivo and mass production. We generated tetravalent anti-Tie2 antibodies by fusing two Fab regions head-to-tail ${ }^{26,27}$. Lutikizumab (ABT-981), a dual variable domain that simultaneously binds IL- $1 \alpha$ and IL-1 $1 \beta$, was developed using the same format. In a clinical study, lutikizumab was well tolerated and behaved similarly to conventional monoclonal antibodies with a half-life of 10 to 14 days $^{26}$. We have confirmed that ASP4021 has good pharmacokinetic properties in rats and non-human primates (data not shown), and expect it to also be well tolerated and stable in humans. ASP4021 was efficiently produced using both CHO and 293 cell systems, which are commonly used for mass production of therapeutic antibodies. Thus, ASP4021 overcomes the manufacturability and stability limitations of Ang1.

In previous studies, anti-Tie2 antibodies, 15B8 and 1-4h, showed agonistic activity in the bi-valent antibody form. However, whereas ASP4021 showed cross-reactivity for rodent Tie2, 
neither 15B8 nor 1-4h bound to rodent Tie $2^{15}$, suggesting that the epitope recognized by ASP 4021 on the surface of Tie2 differs from that of the previous antibodies. The epitope recognized by an antibody might be important for its agonistic activity, given that not all antibody clones in our study showed agonistic activity even when genetically engineered into tetra-valent antibodies. We are currently performing detailed analysis of the epitope of ASP4021. We expect that the epitope of ASP4021 will be similar to the binding site of Ang1 on Tie2, because its clonal prototype 2-16 competed with laveled-Ang1 in the antibody screening step. Further, like Ang1, Ang2 also interacts exclusively with the Ig2 domain of Tie2 in a similar manner ${ }^{28,29}$. Therefore, ASP4021 likely competes against Ang2, a natural antagonist of Tie2 that is involved in vascular leakage and abnormal vessel structure and is elevated in the plasma of diabetic retinopathy patients ${ }^{8}$, making it a potential therapeutic target for retinal disease. Several drug candidates targeting Ang2 are under investigation in clinical trials. Faricimab, a bispecific antibody that inhibits VEGF-A and Ang2, is in phase 3 trials for neovascular age-related macular degeneration and diabetic macular edema ${ }^{30}$. Nesvacumab, a monoclonal antibody that inhibits Ang2, was examined in combination with aflibercept to determine the potential for additional benefits over aflibercept monotherapy in phase 2 studies; however, the trial failed ${ }^{30}$. Because the plasma concentration of Ang2 raises at the ischemic disease condition, it might be difficult for Ang2 targeting drugs to activate the Tie-2/angiopoietin 
pathway stably. ASP4021 can modulate the Tie2/angiopoietin pathway directly and its effect might be unaffected by the concentration of Ang2. We think our approach is the best way to treat the ischemic diseases.

To investigate the efficacy of ASP4021 in these diseases, we are conducting evaluations in a pericyte loss model as a disease model of diabetic retinopathy and diabetic macular edema. These results are expected to pave the way for new treatment methods for diseases associated with vascular weakness such as diabetic retinopathy, diabetic macular edema and critical limb ischemia. 


\section{ONLINE METHODS}

Ethics statement

All methods were carried out in accordance with the institutional guidelines approved by Astellas Pharma as well as relevant guidelines and regulations. All animal experiments were approved by the Institutional Animal Care and Use Committee (IACUC) of Astellas Pharma and performed in accordance with the guidelines of the Care and Use of Laboratory Animals at Astellas Pharma as well as in full compliance of the ARRIVE guidelines. Tsukuba Research Center was awarded Accreditation Status by the AAALAC International.

Animals

SD rats (male, 4-5-weeks-old) were obtained from Charles River Laboratories (Japan).

Cell culture

The human Tie2-expressing mouse pro-B cell line Ba/F3 was established by Dr. Nobuyuki Takakura ${ }^{31}$ and cultured in RPMI1640 supplemented 10\% fetal bovine serum (FBS), 1\% penicillin-streptomycin and $1 \%$ mouse IL-3 culture supplement. Another human Tie2-expressing $\mathrm{Ba} / \mathrm{F} 3$ cell line was 
established at Astellas Pharma by transfecting a human Tie2-expression plasmid into Ba/F3 (RIKEN;

RCB0805), selecting using blasticidin, and culturing in RPMI1640 supplemented with 10\% FBS, 1\% penicillin-streptomycin, $1 \%$ mouse IL-3 culture supplement and $12 \mu \mathrm{g} / \mathrm{mL}$ blasticidin. Human umbilical vein endothelial cells (HUVEC) were purchased from Lonza (C2517AS; Lot. No. 8F3288) and cultured in EGM-2 basal medium containing growth factors (Lonza; CC-3162).

Immunization and screening of anti-Tie2 antibodies

Monoclonal antibodies against Tie2 were produced by immunizing VelocImmune mice (Regeneron), which are genetically modified to produce human antibodies. For immunization, a recombinant human Tie2-Fc chimeric protein (R\&D Systems) was injected into the VelocImmune mice together with an adjuvant for causing an immune reaction. Using a standard method, the lymph nodes of the immunized mice were extracted, and the lymphocytes were collected and cell-fused with mouse-derived myeloma cell SP2/0 (ATCC; CRL-1581), to prepare a hybridoma. The hybridoma was cloned by limiting dilution and each clone was cultured in CD Hybridoma Medium (Thermo Fisher Scientific), a serumfree culture medium. The antibodies were purified from the culture supernatant using a Protein G Column (GE Healthcare). For SEC analysis, purified antibody solutions were fractionated by size exclusion chromatography using TSK-GEL G3000SW (TOSOH; 5789) equilibrated with PBS. The 
binding of each antibody to human, monkey, rat, and mouse Tie 2 was evaluated by cell ELISA assay using $\mathrm{CHO}$ cells expressing each Tie 2 protein. Competitive binding activity of the test antibody to Tie2 was evaluated against COMP-Ang1 using competitive ELISA. Briefly, an expression vector containing COMP-Ang1 was introduced into HEK293 cells. COMP-Ang1 was purified from the culture supernatant and biotin-labeled and mixed with the purified antibody, and the mixture was added to a plate immobilized with recombinant human Tie2-Fc chimeric protein. Streptavidin-labeled HRP followed by TMB color developing reagent (Dako) were added to detect biotin-labeled COMPAng1. To stop the reaction, $2 \mathrm{M}$ sulfuric acid was added. Subsequently, absorbance at $450 \mathrm{~nm}$ was measured to evaluate competitive binding of the test antibody against COMP-Ang1.

Agonistic activity assay

Human Tie2-expressing Ba/F3 cells were suspended in RPMI1640 medium (Thermo Fisher Scientific) supplemented with $0.05 \%$ FBS at $2 \times 10^{5}$ cells $/ \mathrm{mL}$ and added $80 \mu \mathrm{L}$ to each well of a 96 -well plate (Sumitomo Bakelite). Thereafter, $20 \mu \mathrm{L}$ of the purified antibody or Ang 1 diluted with phosphate buffer saline (PBS) was added. After culturing for 72 hours in a $\mathrm{CO}_{2}$ incubator set to $37^{\circ} \mathrm{C}, 50 \mu \mathrm{L}$ of the cell suspension was transferred to a white 96-well plate (Thermo Fisher Scientific). To measure an intracellular ATP using CellTiter-Glo Luminescent Cell Viability Kit (Promega), 50 $\mu \mathrm{L}$ of CellTiter- 
Glo reagent was added into each well. The microplate was shaken and incubated in a dark place.

Luminescence was measured using EnVisionHTS Multilabel Reader (PerkinElmer).

Generation of a genetically engineered fully human tetra-valent antibody

The genes encoding the heavy and light chains of the antibody were cloned from a hybridoma. After sequencing the antibody, the framework region of the light and heavy chains was replaced to improve the antibody's physicochemical properties and stability. To construct an expression vector containing a fully human anti-Tie2 antibody, a gene encoding a signal sequence ${ }^{32}$ and a gene encoding the constant region of human Ig $\gamma 1$ were linked to the 5' and 3' ends, respectively, of the gene encoding the heavy chain variable region, and the heavy chain gene was inserted into a GS vector pEE6.4 (Lonza). Further, a gene encoding a signal sequence and a gene encoding the constant region of the human kappa chain were connected to the 5' and 3' ends, respectively, of the gene encoding the light chain variable region. This light chain gene was inserted into a GS vector pEE12.4 (Lonza). To construct an expression vector containing a tetra-valent antibody, a structure consisting of a heavy chain variable region and a $\mathrm{CH} 1$ region was linked to the $\mathrm{N}$ terminus of the fully human anti-Tie2 
antibody heavy chain with amino acid mutations L234A, L235A and P331S through a linker and inserted into pEE6.4 with a signal sequence. For the light chain, the expression vector for the fully human antibody described above was used. The GS vectors into which the genes of the heavy and light chains of the antibody had been each inserted were digested with restriction enzymes NotI and PvuI, and ligated using a Ligation-Convenience Kit (Nippon Gene) or the ligation reagent, Ligation high Ver. 2 (TOYOBO). To express the antibodies, expression vectors were transfected into FreeStyle 293 cells (Thermo Fisher Scientific), Expi 293 cells (Thermo Fisher Scientific) or CHO-K1SV cells (Lonza) and cultured for 5-7 days. Antibodies were purified from each of the culture supernatants using a Protein A column, a Protein G column or MabSelect SuRe (GE Healthcare) followed by size exclusion chromatography, as described above.

Anti-apoptotic assay

Human Tie2-expressing Ba/F3 cells established at Astellas Pharma were washed three times with medium and suspended in RPMI1640 with GlutaMax and 10\% FBS. A total of $1.6 \times 10^{4}$ cells/ $/ 80 \mu \mathrm{L}$ was added to a 96-well Multi-well Plate for Suspension Culture (Sumitomo Bakelite). ASP4021 or 
Ang1 (R\&D Systems) was serially diluted with PBS, and $20 \mu \mathrm{L}$ of ASP4021 solution, Ang1 solution or PBS alone was added to each well of the culture plate. The culture plate was incubated for 2 days in a $5 \% \mathrm{CO}_{2}$ incubator at $37^{\circ} \mathrm{C}$ and then at room temperature for $10 \mathrm{~min}$. The cells were suspended by pipetting and transferred $(50 \mu \mathrm{L})$ to a 96-well white microplate (Thermo Fisher Scientific). To measure caspase-3 and -7 activity for evaluation of apoptosis, $50 \mu \mathrm{L}$ of 4 -fold diluted Caspase-Glo3/7 assay solution (Promega) with Tris-buffered saline and $10 \mathrm{mmol} / \mathrm{L}$ DTT (Wako), $10 \mathrm{mmol} / \mathrm{L} \mathrm{CDTA}$ (Nacalai Tesqu), 50\% glycerol (Nacalai Tesque) and 5\% TritonX-100 (MP Biomedicals) were added to each well. The microplate was shaken and incubated for $30 \mathrm{~min}$ in the dark. Luminogenic caspase-3/7 substrate, which is proportional to the amount of caspase activity, was measured using an EnVision HTS Multilabel Reader (PerkinElmer).

Phosphorylation of Tie2 in human umbilical vein endothelial cells (HUVEC)

HUVEC were seeded in a $10 \mathrm{~cm}$ dish at $1.5 \times 10^{6}$ cells in EGM-2 basal medium containing growth factors. Cells were washed once with PBS and serum-starved in EGM-2 medium for $2 \mathrm{~h}$ before being exposed to $0.18 \mathrm{nM}(10 \mathrm{ng} / \mathrm{mL})$ of Ang1 or a final concentration of $0.04,0.4$, or $4 \mathrm{nM}(10,100,1000$ $\mathrm{ng} / \mathrm{mL}$ ) of ASP4021 for $30 \mathrm{~min}$. PBS was used as a vehicle control. Following the treatment, cells were washed once with cold PBS and lysed with RIPA buffer (Sigma), $1 \times$ Halt protease and 
phosphatase inhibitor (Thermo Fisher Scientific), and $50 \mathrm{U} / \mathrm{mL}$ benzonase nuclease (Merck Millipore).

After 30 minutes on ice, lysates were centrifuged, and the supernatant was collected. Protein concentrations in the supernatant were determined using a BCA protein assay kit (Thermo Fisher Scientific). The samples ( $8.5 \mu \mathrm{g}$ protein/lane) were reduced and separated by electrophoresis, before being transferred onto an Invitrolon PVDF sheet (Thermo Fisher Scientific). After blocking with Blocking One (Nacalai Tesque), each membrane was incubated with antibodies against Tie2 (Cell Signaling Technology; \#4224), pTie2 (Abcam; ab151704) or actin (Abcam; AB8227) at $4^{\circ} \mathrm{C}$ overnight. After washing, membranes were incubated with an anti-rabbit (GE Healthcare) or anti-mouse $\operatorname{IgG}$ HRP-linked antibody (Thermo Fisher Scientific) and each protein was detected using the chemiluminescence reagent Clarity Western ECL substrate (Bio-Rad) with a CCD camera (Bio-Rad; ChemiDoc). Signal intensity was quantified using Image Labs Software v5.2 (Bio-Rad). The assay was performed once in duplicate. The signal intensity for pTie2 was normalized to that for Tie2. Mean values of pTie 2 in each concentration group relative to those of the vehicle control group were calculated from the normalized pTie2 values of each sample.

\section{Direct ELISA}

A recombinant human Tie1-Fc chimeric protein or human Tie2-Fc chimeric protein (R\&D Systems) 
was prepared in PBS, added to a microplate and incubated at $4^{\circ} \mathrm{C}$ overnight. The immobilized solution was removed, and 20\% Blocking One (Nacalai Tesque) containing Tris-buffered saline with $0.05 \%$ Tween (TBS-T) was added and left to stand at room temperature for 1 hour. ASP4021 diluted with 5\% Blocking One containing TBS-T was added and incubated at room temperature for 1.5 hours, and then washed with TBS-T. A goat anti-human kappa-HRP (Southern Biotech) secondary antibody was added and incubated at room temperature for 1 hour, before washing with TBS-T. TMB + One-Step Substrate System (Agilent) was then added to each well. The solution turned blue after incubating for about 20 to $30 \mathrm{~min}$. To stop the reaction, $1 \mathrm{~mol} / \mathrm{L}$ sulfuric acid was added to each well, turning the solution yellow. Absorbance at $450 \mathrm{~nm}$ was measured using Infinite M200 Pro (Tecan).

Biacore analysis

Recombinant human, rat and mouse Tie2-Fc chimeric protein (R\&D Systems), recombinant monkey Tie2-Fc chimeric protein (Sino Biological) were each immobilized on a sensor chip CM5 (GE Healthcare) using Amine Coupling Kit (GE Healthcare) and HBS-EP+ buffer (GE Healthcare) in accordance with the manufacturer's instructions. On the blank cell (flow cell 1), the injection of antigen solution was omitted. Measurement was performed three times each with four types of antigens using Biacore T200 (GE Healthcare). The kinetic data were obtained by injecting increasing 
concentration of antibody into the sensor chip at a flow rate of $50 \mu \mathrm{l} / \mathrm{min}$. The measurements were carried out in HBS-EP+ buffer. Contact time and dissociation time were $120 \mathrm{sec}$ and $300 \mathrm{sec}$, respectively. Data analysis was performed with Biacore T200 evaluation software version 3.0 (GE Healthcare) by fitting the results to sensorgrams obtained from a bivalent binding model.

Pharmacokinetic assay

ASP4021 was subcutaneously administered to SD rats. Blood was collected from each rat at 8 and 26 hours, and 2, 3, 5 and 7 days after administration of the antibody, and plasma was separated. The concentration of ASP4021 in plasma was measured using human Tie2-Fc direct ELISA.

Mustard oil-induced vascular permeability model

TIE-1-Ig 1 -WT, 2-16A2 or ASP4021 diluted with PBS was subcutaneously administered to SD rats. At 48 hours after administration of the antibody, Evans blue dye dissolved in physiological saline (Sigma) was intravenously administered. Immediately, $20 \mu \mathrm{l}$ of allyl isothiocyanate (also known as mustard oil; Nacalai Tesque) diluted with 5\% mineral oil (Sigma) was applied to one ear, and mineral oil to the contralateral ear. After 30 minutes, both ears were sampled, weighed, then immersed in $1 \mathrm{~mL}$ 
of formamide, and incubated at $70^{\circ} \mathrm{C}$ overnight to extract Evans blue dye from the ear tissue. The Evans blue dye concentration was determined from the absorbance (measurement wavelength of 620 $\mathrm{nm}$ and control wavelength of $740 \mathrm{~nm}$ ) of the extract. The amount of leakage per ear weight was calculated by dividing the Evans blue dye concentration by the weight of the ear. The final amount of leaked Evans Blue dye from each animal was calculated by subtracting the amount of leaked Evans blue dye from the ear that received mineral oil from the amount from the ear that received mustard oil in the same animal. The amount of leaked Evans blue dye was used as an index of vascular permeability.

Statistical analyses

Data are presented as mean \pm SD or mean \pm SEM. In the mustard oil-induced vascular permeability model, significant differences between the test and vehicle groups were determined using Student's ttest or Dunnett's multiple comparisons test by using GraphPad Prism. P $<0.05$ was considered statistically significant. 


\section{Acknowledgements}

The authors wish to thank Yoshinori Ishii for knowledgeable discussions and insight into the analysis and generation of the engineered antibody; Takao Suzuki for data acquisition and analysis; and Shiomi Nagasawa and Akira Sakamoto for technical assistance.

This research was financially supported by Astellas Pharma Inc.

\section{Author Contributions}

Y.K. contributed to data acquisition, analysis and wrote the manuscript.

H.N. designed and conducted the research, especially the in vivo study, contributed to data acquisition and analysis, and helped to draft the manuscript.

S.Y. designed and conducted research mainly related to the generation of the engineered antibody, contributed to data acquisition and analysis.

C.U. contributed to data acquisition and analysis.

M.K. designed and conducted the research, contributed to data acquisition and analysis, and helped to draft the manuscript.

All authors contributed to and approved the final version of the manuscript. 


\section{Competing Interests Statement}

The authors are all employees of Astellas Pharma Inc. Astellas Pharma Inc. holds patents (PCT/JP2015/070089) on Novel Anti-Human Tie2 Antibody. H.N., S.Y. and M.K. are the inventor of the patent. 


\section{References}

1. Kim, I. et al. Angiopoietin-1 regulates endothelial cell survival through the phosphatidylinositol 3'-Kinase/Akt signal transduction pathway. Circulation research 86, 24-29 (2000).

2. Gavard, J., Patel, V. \& Gutkind, J.S. Angiopoietin-1 Prevents VEGF-Induced Endothelial Permeability by Sequestering Src through mDia. Developmental Cell 14, 25-36 (2008).

3. Thurston, G. et al. Leakage-Resistant Blood Vessels in Mice Transgenically Overexpressing Angiopoietin-1. Science 286, 2511-2514 (1999).

4. Suri, C. et al. Requisite Role of Angiopoietin-1, a Ligand for the TIE2 Receptor, during Embryonic Angiogenesis. Cell 87, 1171-1180 (1996).

5. Alfieri, A. et al. Angiopoietin-1 regulates microvascular reactivity and protects the microcirculation during acute endothelial dysfunction: role of eNOS and VE-cadherin. Pharmacological research 80, 43-51 (2014).

6. Uemura, A. et al. Recombinant angiopoietin-1 restores higher-order architecture of growing blood vessels in mice in the absence of mural cells. The Journal of clinical investigation 110, 1619-1628 (2002).

7. Joussen, A.M. et al. Suppression of diabetic retinopathy with angiopoietin-1. The American journal of pathology 160, 1683-1693 (2002).

8. Lip, P.L. et al. Plasma vascular endothelial growth factor, angiopoietin-2, and soluble angiopoietin receptor tie-2 in diabetic retinopathy: effects of laser photocoagulation and angiotensin receptor blockade. Br J Ophthalmol 88, 1543-1546 (2004).

9. Huang, J. et al. Sendai viral vector mediated angiopoietin-1 gene transfer for experimental ischemic limb disease. Angiogenesis 12, 243-249 (2009).

10. Brandão, D., Costa, C., Canedo, A., Vaz, G. \& Pignatelli, D. Endogenous vascular endothelial growth factor and angiopoietin-2 expression in critical limb ischemia.

International angiology : a journal of the International Union of Angiology 30, 25-34 (2011).

11. Oh, N. et al. A Designed Angiopoietin-1 Variant, Dimeric CMP-Ang1 Activates Tie2 and Stimulates Angiogenesis and Vascular Stabilization in N-glycan Dependent Manner. Scientific reports 5, 15291 (2015).

12. Cho, C.H. et al. Long-term and sustained COMP-Ang1 induces long-lasting vascular 
enlargement and enhanced blood flow. Circulation research 97, 86-94 (2005).

13. Cho, C.-H. et al. COMP-Ang1: A designed angiopoietin-1 variant with nonleaky angiogenic activity. Proceedings of the National Academy of Sciences of the United States of America 101, 5547-5552 (2004).

14. Hansbury, M.J., Nicosia, R.F., Zhu, W.-H., Holmes, S.J. \& Winkler, J.D. Production and characterization of a Tie2 agonist monoclonal antibody. Angiogenesis 4, 29-36 (2001).

15. Hwang, B. et al. Stimulation of angiogenesis and survival of endothelial cells by human monoclonal Tie2 receptor antibody. Biomaterials 51, 119-128 (2015).

16. Maisonpierre, P.C. et al. Angiopoietin-2, a natural antagonist for Tie2 that disrupts in vivo angiogenesis. Science 277, 55-60 (1997).

17. Fekete, S., Beck, A., Veuthey, J.-L. \& Guillarme, D. Theory and practice of size exclusion chromatography for the analysis of protein aggregates. Journal of Pharmaceutical and Biomedical Analysis 101, 161-173 (2014).

18. $\mathrm{Xu}, \mathrm{D}$. et al. In vitro characterization of five humanized OKT3 effector function variant antibodies. Cellular immunology 200, 16-26 (2000).

19. Hezareh, M., Hessell, A.J., Jensen, R.C., van de Winkel, J.G.J. \& Parren, P.W.H.I. Effector Function Activities of a Panel of Mutants of a Broadly Neutralizing Antibody against Human Immunodeficiency Virus Type 1. Journal of Virology 75, 12161-12168 (2001).

20. Oganesyan, V., Gao, C., Shirinian, L., Wu, H. \& Dall'Acqua, W.F. Structural characterization of a human $\mathrm{Fc}$ fragment engineered for lack of effector functions. Acta crystallographica. Section D, Biological crystallography 64, 700-704 (2008).

21. Idusogie, E.E. et al. Mapping of the C1q Binding Site on Rituxan, a Chimeric Antibody with a Human IgG1 Fc. The Journal of Immunology 164, 4178 (2000).

22. Liu, L. Pharmacokinetics of monoclonal antibodies and Fc-fusion proteins. Protein \& Cell 9, 15-32 (2018).

23. Miles, A.A. \& Miles, E.M. Vascular reactions to histamine, histamine-liberator and leukotaxine in the skin of guinea-pigs. J Physiol 118, 228-257 (1952).

24. Thurston, $\mathrm{G}$. et al. Angiopoietin-1 protects the adult vasculature against plasma leakage. Nature Medicine 6, 460-463 (2000).

25. Davis, S. et al. Angiopoietins have distinct modular domains essential for receptor binding, dimerization and superclustering. Nature structural biology 10, 38-44 (2003).

26. Kosloski, M.P. et al. Pharmacokinetics and Tolerability of a Dual Variable Domain Immunoglobulin ABT-981 Against IL-1 $\alpha$ and IL-1 $\beta$ in Healthy Subjects and Patients With Osteoarthritis of the Knee. Journal of clinical pharmacology 56, 1582-1590 (2016). 
27. Miller, K. et al. Design, Construction, and In Vitro Analyses of Multivalent Antibodies. The Journal of Immunology 170, 4854-4861 (2003).

28. $\mathrm{Yu}, \mathrm{X}$. et al. Structural basis for angiopoietin-1-mediated signaling initiation. Proceedings of the National Academy of Sciences 110, 7205-7210 (2013).

29. Barton, W.A. et al. Crystal structures of the Tie2 receptor ectodomain and the angiopoietin-2-Tie2 complex. Nature structural \& molecular biology 13, 524-532 (2006).

30. Hussain, R.M., Neiweem, A.E., Kansara, V., Harris, A. \& Ciulla, T.A. Tie-2/Angiopoietin pathway modulation as a therapeutic strategy for retinal disease. Expert Opinion on Investigational Drugs 28, 861-869 (2019).

31. Takakura, N. et al. Critical role of the TIE2 endothelial cell receptor in the development of definitive hematopoiesis. Immunity 9, 677-686 (1998).

32. Whittle, N. et al. Expression in COS cells of a mouse-human chimaeric B72.3 antibody. Protein Engineering, Design and Selection 1, 499-505 (1987). 


\section{Figure legends}

Fig. 1

a

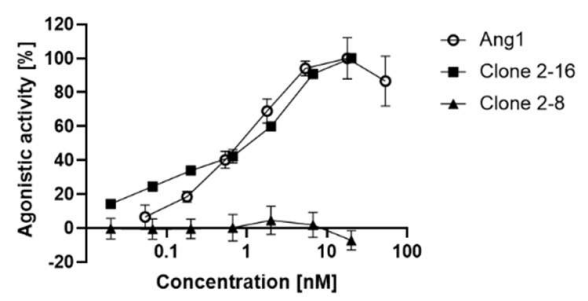

d

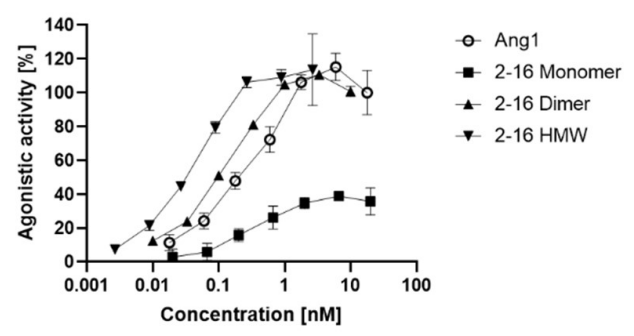

b

Fraction number

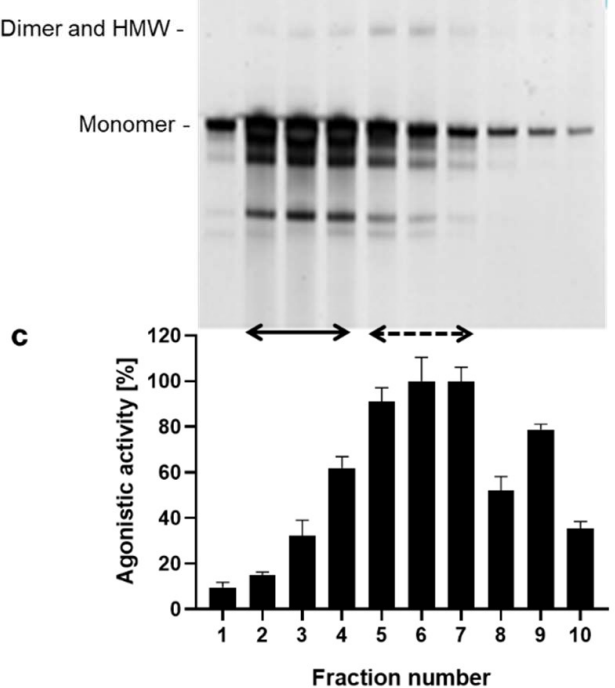

Fig. 1| Characterization of newly identified anti-Tie2 agonistic antibodies. a, Agonistic activity of anti-Tie2 antibodies produced by hybridoma clones 2-16 and 2-8 were assessed in human Tie2expressing $\mathrm{Ba} / \mathrm{F} 3$ compared to Ang1. Agonistic activity (\%) was determined using a CellTiter-Glo assay, with basal activity without any ligand (PBS) defined as $0 \%$, and the activity of $17.9 \mathrm{nM}(1$ $\mu \mathrm{g} / \mathrm{mL}$ ) Ang1 defined as 100\%. Data represent mean \pm SEM. b,c, Fractionation of the antibody solution using SEC. b shows SDS PAGE of each fraction, and c shows the agonistic activity of each 
fraction in human Tie2-expressing Ba/F3. Solid (left) arrow indicates the monomer-rich fractions, dashed (right) arrow indicates the fractions with higher agonistic activity. Agonistic activity (\%) was determined using a CellTiter-Glo assay, with basal activity without any ligand (PBS) defined as $0 \%$, and the activity of fraction 6 defined as 100\%. d, Agonistic activity of purified 2-16 monomer, dimer and HMW human monoclonal antibodies in human Tie2-expressing $\mathrm{Ba} / \mathrm{F} 3$ compared to Ang1. Agonistic activity (\%) was determined as Fig.1a. 
Fig. 2

a

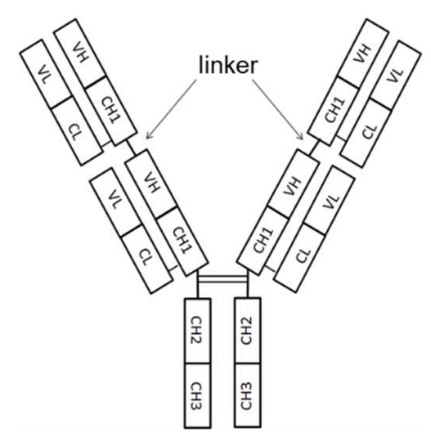

C
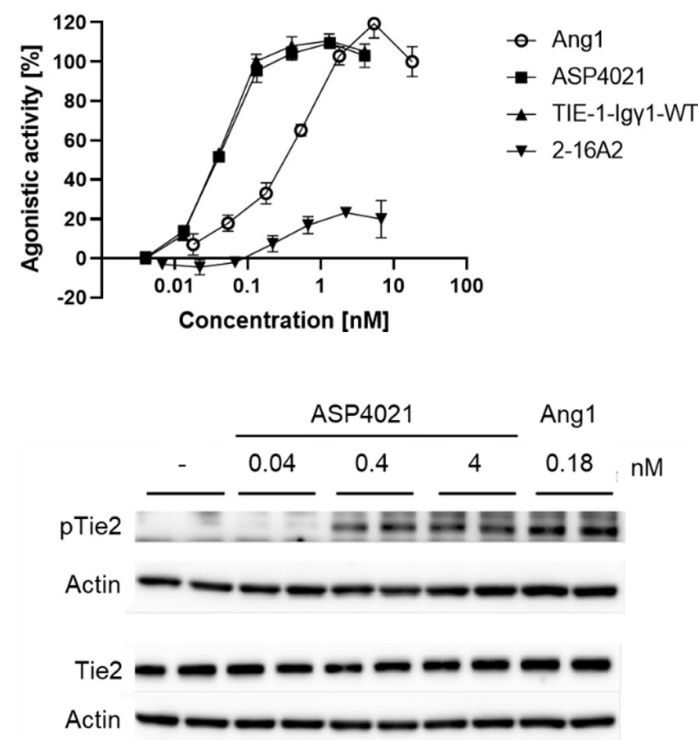

b

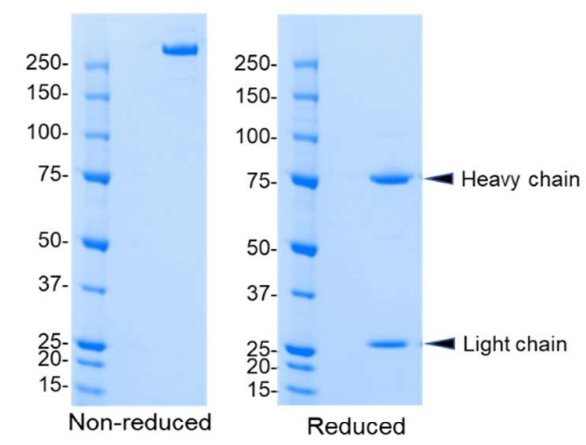

d

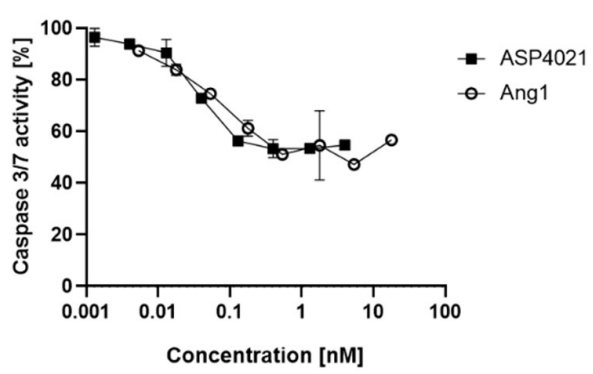

Fig. 2| Generation and evaluation of the tetra-valent anti-human Tie2 antibody. a, Structure of the tetra-valent antibody. b, SDS-PAGE of the tetra-valent antibody under non-reduced (left) and reduced (right) conditions. Molecular weights $(\mathrm{kDa})$ are indicated on the left of each gel. c, Agonistic 
activity of the tetra-valent and bi-valent antibodies in human Tie2-expressing $\mathrm{Ba} / \mathrm{F} 3$ compared to Ang1.

Agonistic activity (\%) was determined as Fig.1a. d, Anti-apoptotic activity of ASP4021 in human Tie2-expressing $\mathrm{Ba} / \mathrm{F} 3$ compared to Ang1. Caspase 3/7 activity (\%) was calculated using the Caspase-

Glo 3/7 assay, with basal activity with IL-3 defined as $0 \%$, and activity without any ligand (PBS) defined as $100 \%$. Data represent mean \pm SEM. e, Phosphorylation of Tie2 induced by ASP4021 in HUVEC compared to Ang1. HUVEC were treated with ASP4021 or recombinant Ang1 for $30 \mathrm{~min}$. Levels of phosphorylated Tie2, total Tie2 and actin in cell lysates were detected using Western blotting. The assay was performed in duplicate. Full-size versions of Western blots are depicted in Supplementary Figure S1. 
Fig. 3

a

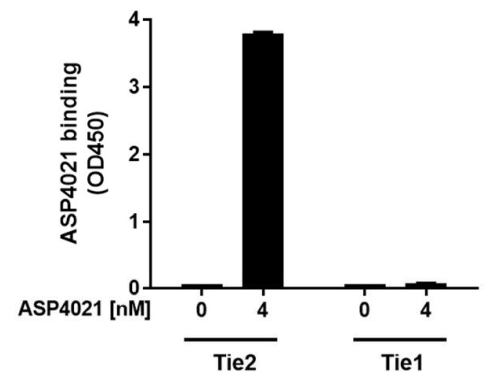

C

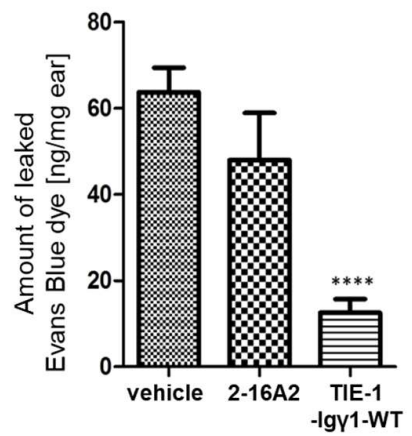

b

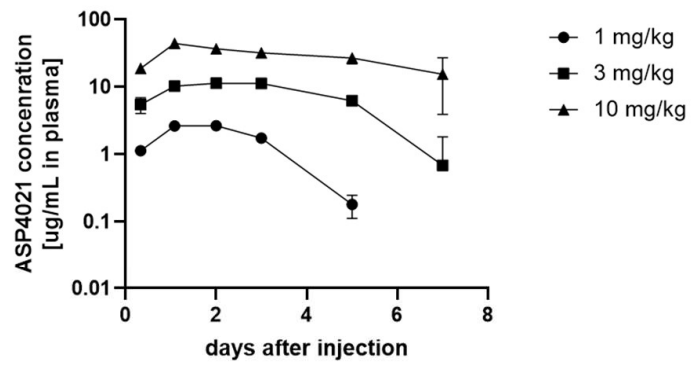

d

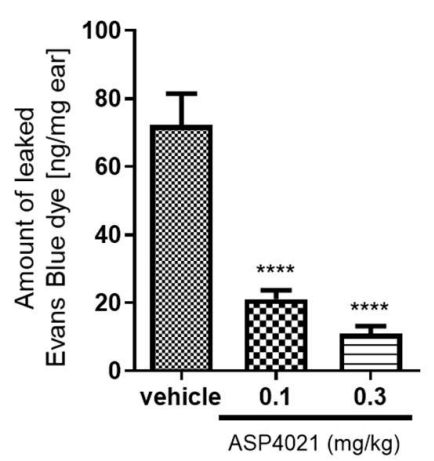

Fig. 3| Effect of ASP4021 and related antibodies on vascular permeability in vitro and in vivo. a,

Binding activity of ASP4021 to human Tie2 and Tie1 by direct ELISA. ASP4021 binding is shown as the absorbance at OD450. Each value is expressed as mean \pm SEM. b, Pharmacokinetics analysis of ASP4021 in rats. The concentration of ASP4021 in rat plasma after subcutaneous administration was measured using an ELISA-based assay. Each value indicates the mean $\pm \mathrm{SD}$ of 3 rats per group. c, Inhibitory effect of 2-16A2 and TIE-1-Ig $\gamma 1-W T$ on vascular permeability in a rat mustard oil-induced vascular permeability model following treatment with $0.3 \mathrm{mg} / \mathrm{kg}$. The vertical axis indicates the 
amount of leaked Evans Blue dye. The graph indicates the mean \pm SD of 6 rats per group. Student's ttest was employed for statistical analysis. $* * * * p<0.0001$ vs the vehicle group. d, Inhibitory effect of ASP4021 on vascular permeability in a rat mustard oil-induced vascular permeability model following treatment with 0.1 or $0.3 \mathrm{mg} / \mathrm{kg}$. Each graph indicates the mean $\pm \mathrm{SD}$ of 6 rats per group. Dunnet's multiple comparisons test was employed for statistical analysis. ${ }^{* * *} \mathrm{p}<0.0001$ vs the vehicle group. 


\section{Figures}

a

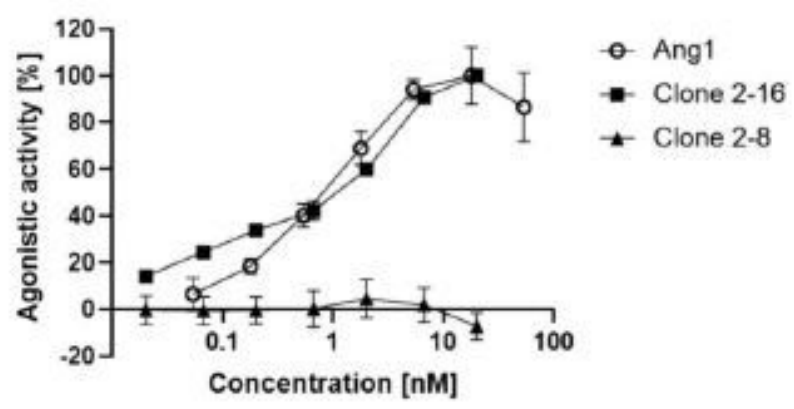

d

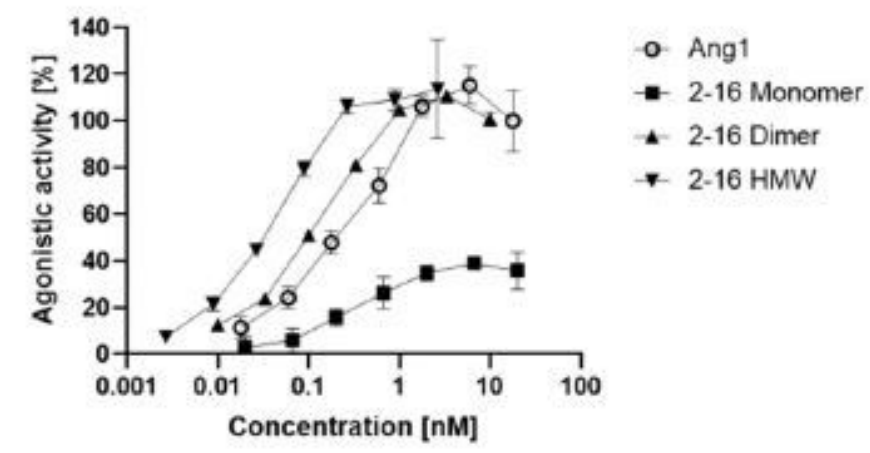

b

Fraction number
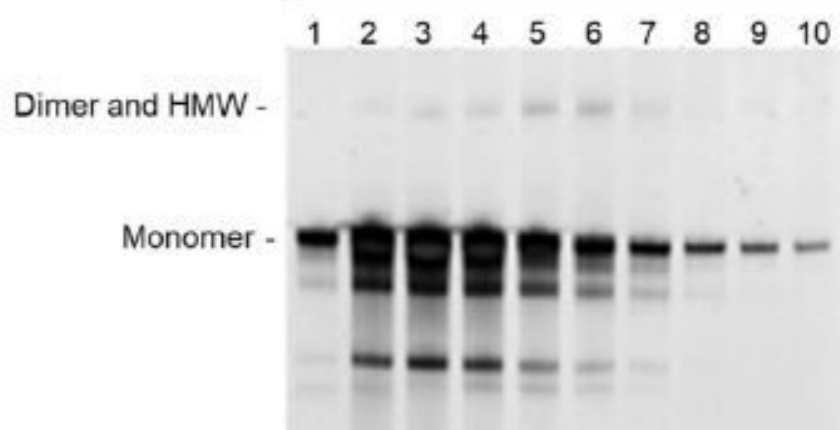

C

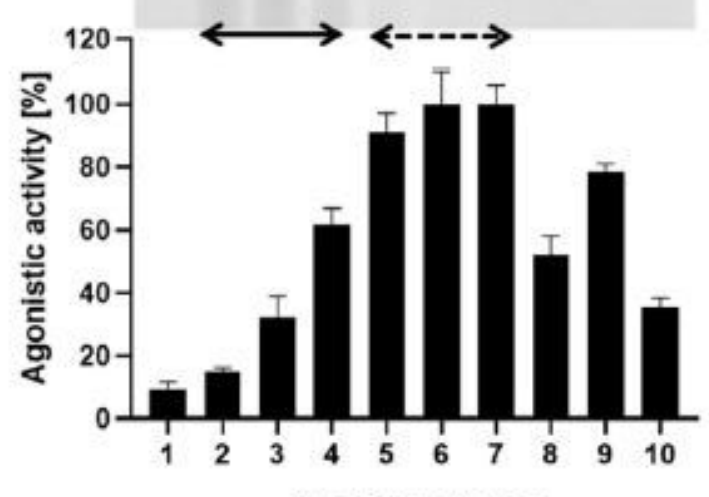

Fraction number

\section{Figure 1}

Characterization of newly identified anti-Tie2 agonistic antibodies. a, Agonistic activity of anti-Tie2 antibodies produced by hybridoma clones 2-16 and 2-8 were assessed in human Tie2- expressing Ba/F3 compared to Ang1. Agonistic activity (\%) was determined using a CellTiter-Glo assay, with basal activity without any ligand (PBS) defined as $0 \%$, and the activity of $17.9 \mathrm{nM}(1 \mu \mathrm{g} / \mathrm{mL})$ Ang1 defined as $100 \%$. Data represent mean \pm SEM. $b, c$, Fractionation of the antibody solution using SEC. $b$ shows SDS PAGE of each fraction, and c shows the agonistic activity of each 30 fraction in human Tie2-expressing Ba/F3. Solid (left) arrow indicates the monomer-rich fractions, dashed (right) arrow indicates the fractions with higher agonistic activity. Agonistic activity (\%) was determined using a CellTiter-Glo assay, with basal activity without any ligand (PBS) defined as $0 \%$, and the activity of fraction 6 defined as $100 \%$. d, Agonistic activity of purified 2-16 monomer, dimer and HMW human monoclonal antibodies in human Tie2-expressing Ba/F3 compared to Ang1. Agonistic activity (\%) was determined as Fig.1a. 
a

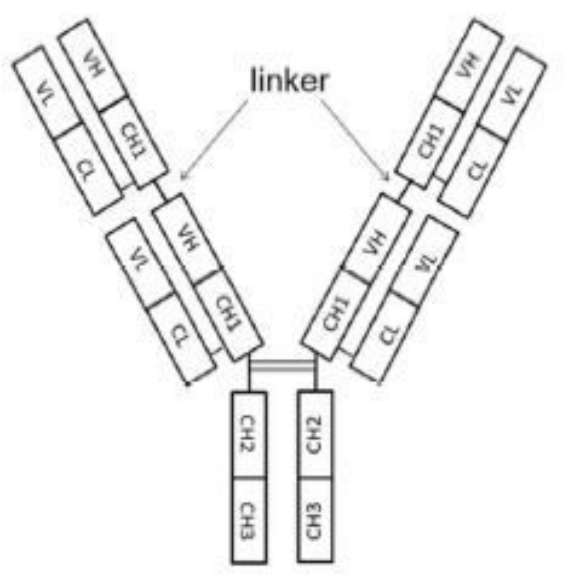

C

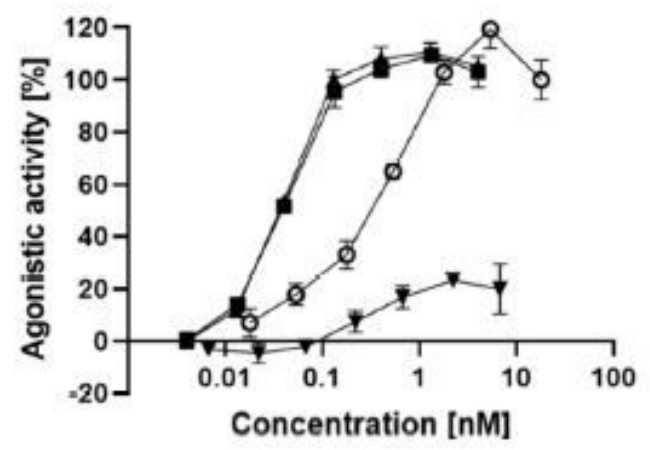

e

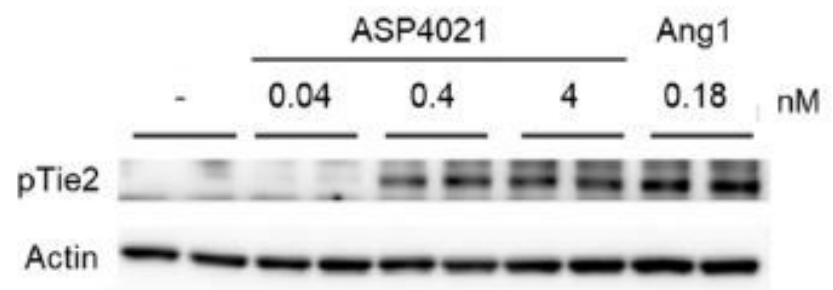

b

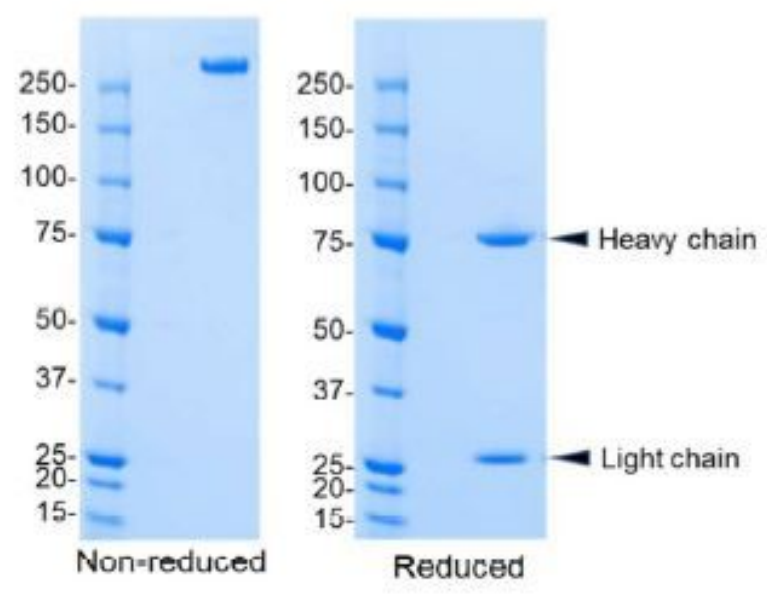

d

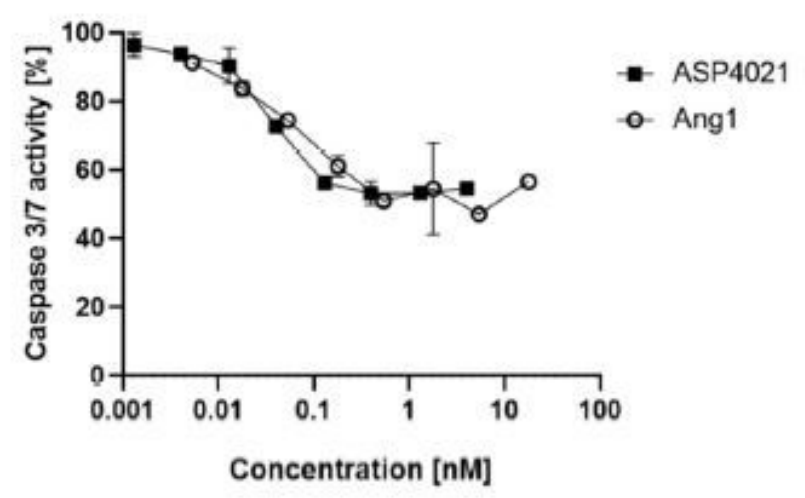

\section{Figure 2}

Generation and evaluation of the tetra-valent anti-human Tie2 antibody. a, Structure of the tetra-valent antibody. b, SDS-PAGE of the tetra-valent antibody under non-reduced (left) and reduced (right) conditions. Molecular weights (kDa) are indicated on the left of each gel. c, Agonistic 32 activity of the tetra-valent and bi-valent antibodies in human Tie2-expressing Ba/F3 compared to Ang1. Agonistic activity (\%) was determined as Fig.1a. d, Anti-apoptotic activity of ASP4021 in human Tie2-expressing $\mathrm{Ba} / \mathrm{F} 3$ compared to Ang1. Caspase 3/7 activity (\%) was calculated using the Caspase- Glo 3/7 assay, with basal activity with IL-3 defined as $0 \%$, and activity without any ligand (PBS) defined as 100\%. Data represent mean \pm SEM. e, Phosphorylation of Tie2 induced by ASP4021 in HUVEC compared to Ang 1. 
HUVEC were treated with ASP4021 or recombinant Ang1 for 30 min. Levels of phosphorylated Tie2, total Tie2 and actin in cell lysates were detected using Western blotting. The assay was performed in duplicate. Full-size versions of Western blots are depicted in Supplementary Figure S1.

a

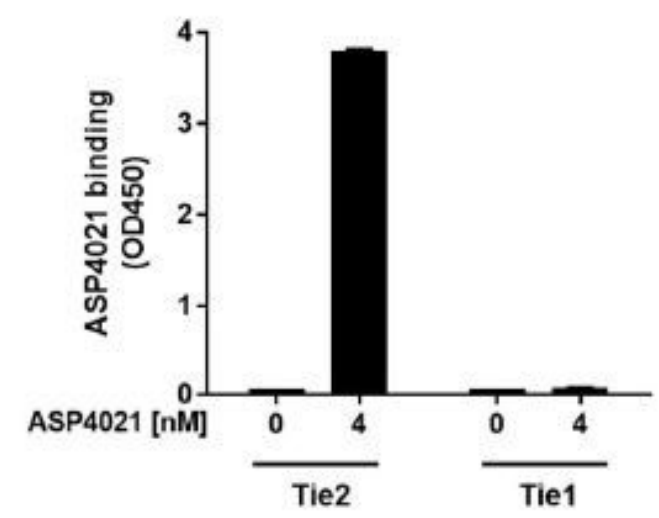

C

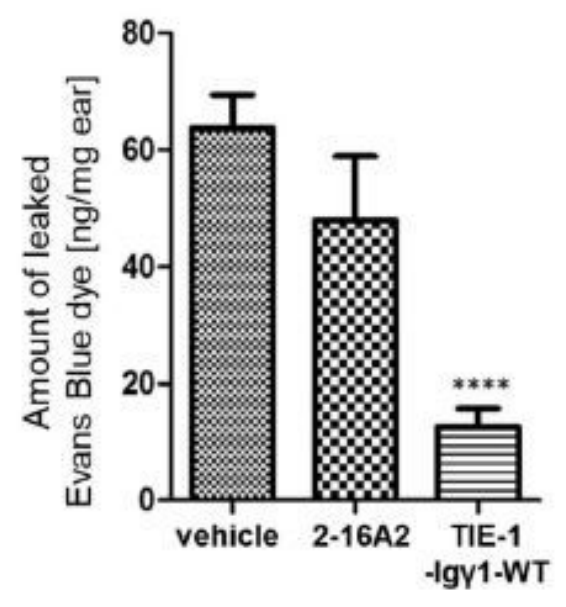

b
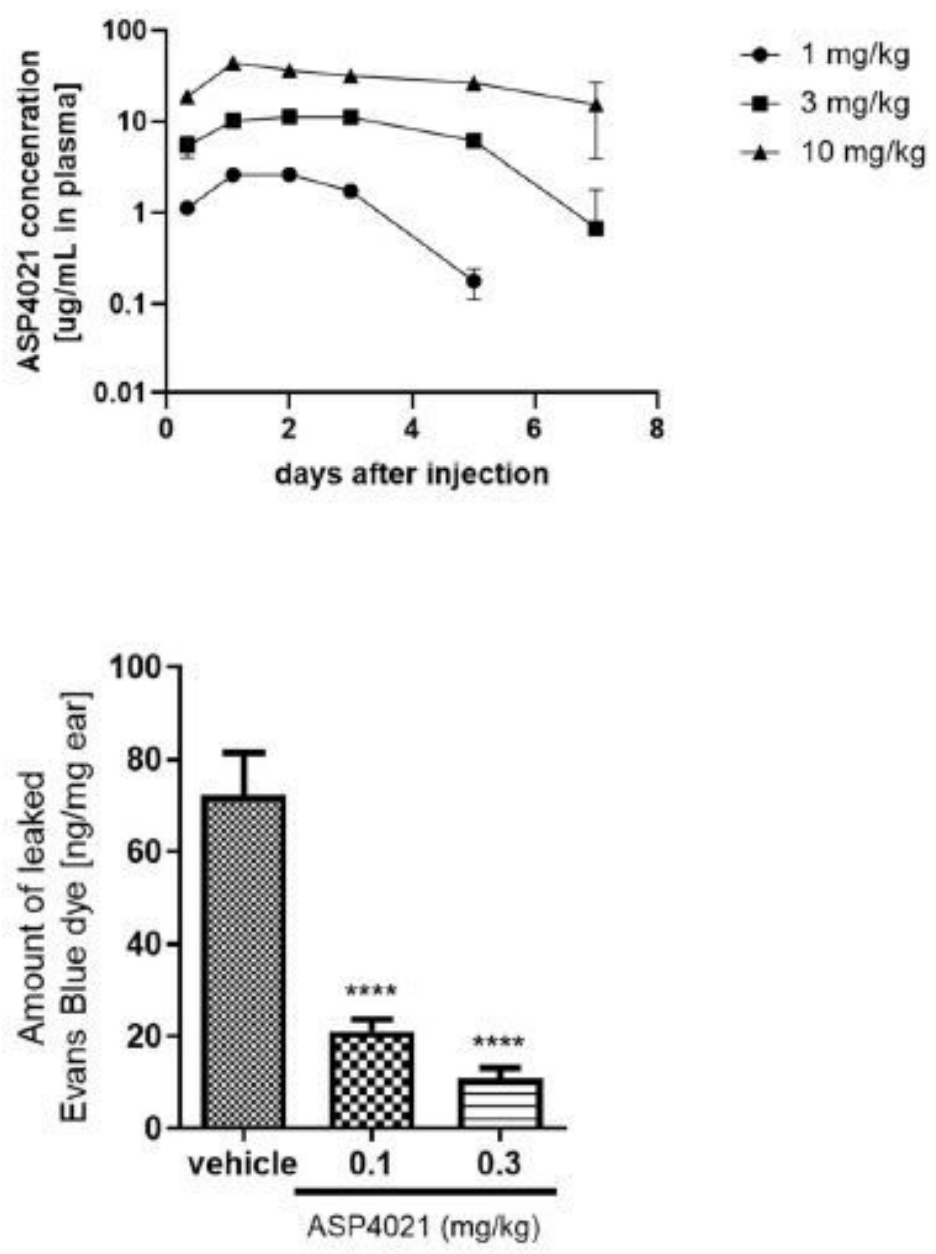

Figure 3

Effect of ASP4021 and related antibodies on vascular permeability in vitro and in vivo. a, Binding activity of ASP4021 to human Tie2 and Tie1 by direct ELISA. ASP4021 binding is shown as the absorbance at OD450. Each value is expressed as mean \pm SEM. $b$, Pharmacokinetics analysis of ASP4021 in rats. The concentration of ASP4021 in rat plasma after subcutaneous administration was measured using an ELISA-based assay. Each value indicates the mean \pm SD of 3 rats per group. c, Inhibitory effect of 2-16A2 and TIE-1-IgY1-WT on vascular permeability in a rat mustard oil-induced vascular permeability model following treatment with $0.3 \mathrm{mg} / \mathrm{kg}$. The vertical axis indicates the 34 amount of leaked Evans Blue dye. The graph indicates the mean \pm SD of 6 rats per group. Student's ttest was employed for statistical analysis. ${ }^{* \star \star \star} p<0.0001$ vs the vehicle group. $d$, Inhibitory effect of ASP4021 on vascular permeability in a rat mustard oil-induced vascular permeability model following treatment with $0.1 \mathrm{or} 0.3 \mathrm{mg} / \mathrm{kg}$. Each 
graph indicates the mean \pm SD of 6 rats per group. Dunnet's multiple comparisons test was employed for statistical analysis. ${ }^{\star \star \star \star} p<0.0001$ vs the vehicle group.

\section{Supplementary Files}

This is a list of supplementary files associated with this preprint. Click to download.

- Supplementaryinformation.pdf 